\title{
Pulse Coupled Neural Networks for Automatic Urban Change Detection at Very High Spatial Resolution
}

\author{
Fabio Pacifici ${ }^{1}$ and William J. Emery ${ }^{2}$ \\ 1 Department of Computer, Systems and Production Engineering, \\ Tor Vergata University, Rome, Italy \\ 2 Department of Aerospace Engineering Science, \\ University of Colorado at Boulder, CO - USA \\ f.pacifici@disp.uniroma2.it
}

\begin{abstract}
In this paper, a novel unsupervised approach based on PulseCoupled Neural Networks (PCNNs) for image change detection is discussed. PCNNs are based on the implementation of the mechanisms underlying the visual cortex of small mammals and with respect to more traditional neural networks architectures own interesting advantages. In particular, they are unsupervised and context sensitive. The performance of the algorithm has been evaluated on very high spatial resolution QuickBird and WorldView-1 images. Qualitative and more quantitative results are discussed.
\end{abstract}

Keywords: Change detection, Pulse Coupled Neural Networks, Urban Environment.

\section{Introduction}

World population growth affects the environment through the swelling of the population in urban areas and by increasing the total consumption of natural resources. Monitoring these changes timely and accurately might be crucial for the implementation of effective decision-making processes. In this context, the contribution of satellite and airborne sensors might be significant for updating land-cover and land-use maps. Indeed, the recent commercial availability of very high spatial resolution visible and near-infrared satellite data has opened a wide range of new opportunities for the use of Earth-observing satellite data. In particular, new systems such as the latest WorldView-1, characterized by the highest spatial resolution, now provide additional data along with very high spatial resolution platforms, such as QuickBird or IKONOS, which have already been operating for a few years.

If on one side this makes available a large amount of information, on the other side, the need of completely automatic techniques able to manage big data archives is becoming extremely urgent. In fact, supervised methods risk to become unsuitable when dealing with such large amounts of data. This is even

E. Bayro-Corrochano and J.-O. Eklundh (Eds.): CIARP 2009, LNCS 5856, pp. 929-942, 2009.

(C) Springer-Verlag Berlin Heidelberg 2009 
more compelling if applications dedicated to the monitoring of urban sprawl are considered. In these cases, the big potential provided by very high spatial resolution images has to be exploited for analyzing large areas, which would be unfeasible if completely automatic procedures are not taken into account.

Most of the research carried out so far focused on medium or high spatial resolution images, whereas only few studies have addressed the problem of fully automatic change detection for very high spatial resolution images. In this case, several issues have to be specifically considered. The crucial ones include possible misregistrations, shadow, and other seasonal and meteorological effects which add up and combine to reduce the attainable accuracy in the change detection results.

In this paper, a novel neural network approach for the detection of changes in multi-temporal very high spatial resolution images is proposed. Pulse-coupled neural networks are a relatively new technique based on the implementation of the mechanisms underlying the visual cortex of small mammals. The visual cortex is the part of the brain that receives information from the eye. The waves generated by each iteration of the algorithm create specific signatures of the scene which are successively compared for the generation of the change map. The proposed method is completely automated since analyzes the correlation between the time signals associated to the original images. This means that no pre-processing, except for image registration, is required. Furthermore, PCNNs may be implemented to exploit at the same time both contextual and spectral information which make them suitable for processing any kind of sub-meter resolution images.

This paper is organized as follows. Section 2 recalls the PCNN model. The application of the algorithm is described in Section 3. Final conclusions follow in Section 4 .

\section{Pulse Coupled Neural Networks}

Pulse Coupled Neural Networks entered the field of image processing in the nineties, following the publication of a new neuron model introduced by Eckhorn et al. 1. Interesting results have been already shown by several authors in the application of this model in image segmentation, classification and thinning 2] 3], including, in few cases, the use of satellite data [4] [5]. Hereafter, the main concepts underlying the behavior of PCNNs are briefly recalled. For a more comprehensive introduction to image processing using PCNN refer to [6].

\subsection{The Pulse Coupled Model}

A PCNN is a neural network algorithm that, when applied to image processing, yields a series of binary pulsed signals, each associated to one pixel or to a cluster of pixels. It belongs to the class of unsupervised artificial neural networks in the sense that it does not need to be trained. The network consists of nodes with spiking behavior interacting each other within a pre-defined grid. The architecture 


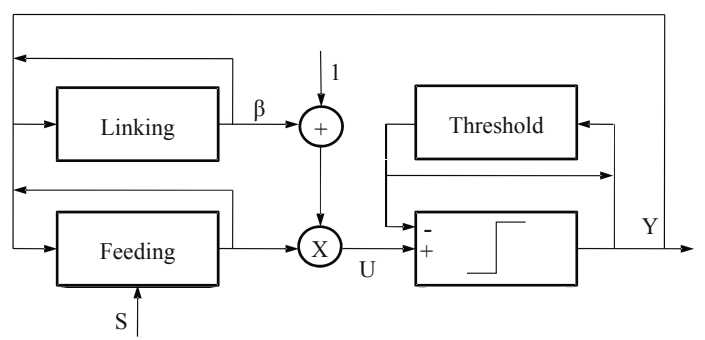

Fig. 1. Schematic representation of a PCNN neuron

of the network is rather simpler than most other neural implementations: there are no multiple layers that pass information to each other. PCNNs only have one layer of neurons, which receives input directly from the original image, and form the resulting pulse image.

The PCNN neuron has three compartments. The feeding compartment receives both an external and a local stimulus, whereas the linking compartment only receives the local stimulus. The third compartment is represented by an active threshold value. When the internal activity becomes larger than the threshold the neuron fires and the threshold sharply increases. Afterwards, it begins to decay until once again the internal activity becomes larger. Such a process gives rise to the pulsing nature of the PCNN.

The schematic representation of a PCNN is shown in Figure 1 while, more formally, the system can be defined by the following expressions:

$$
\begin{gathered}
F_{i j}[n]=e^{-\alpha_{F}} \cdot F_{i j}[n-1]+S_{i j}+V_{F} \sum_{k l} M_{i j k l} Y_{k l}[n-1] \\
L_{i j}[n]=e^{-\alpha_{L}} \cdot L_{i j}[n-1]+V_{L} \sum_{k l} W_{i j k l} Y_{k l}[n-1]
\end{gathered}
$$

where $S_{i j}$ is the input to the neuron $(i j)$ belonging to a $2 \mathrm{D}$ grid of neurons, $F_{i j}$ the value of its feeding compartment and $L_{i j}$ is the corresponding value of the linking compartment. Each of these neurons communicates with neighbouring neurons $(k l)$ through the weights given by $M$ and $W$ respectively. $M$ and $W$ traditionally follow very symmetric patterns and most of the weights are zero. $Y$ indicates the output of a neuron from a previous iteration $[n-1]$. All compartments have a memory of the previous state, which decays in time by the exponent term. The constant $V_{F}$ and $V_{L}$ are normalizing constants. The state of the feeding and linking compartments are combined to create the internal state of the neuron, $U$. The combination is controlled by the linking strength, $\beta$. The internal activity is given by:

$$
U_{i j}[n]=F_{i j}[n]\left\{1+\beta L_{i j}[n]\right\}
$$

The internal state of the neuron is compared to a dynamic threshold, $\Theta$, to produce the output, $Y$, by: 


$$
Y_{i j}[n]= \begin{cases}1 & \text { if } U_{i j}[n]>\Theta_{i j}[n] \\ 0 & \text { otherwise }\end{cases}
$$

The threshold compartment is described as:

$$
\Theta_{i j}[n]=e^{-\alpha_{\Theta}} \cdot \Theta_{i j}[n-1]+V_{\Theta} Y_{i j}[n]
$$

where $V_{\Theta}$ is a large constant generally more than one order of magnitude greater than the average value of $U$.

The algorithm consists of iteratively computing Equation 1 through Equation 5 until the user decides to stop. Each neuron that has any stimulus will fire at the initial iteration, creating a large threshold value. Then, only after several iterations the threshold will be small enough to allow the neuron to fire again. This process is the beginning of the autowaves nature of PCNNs. Basically, when a neuron (or group of neurons) fires, an autowave emanates from that perimeter of the group. Autowaves are defined as normal propagating waves that do not reflect or refract. In other words, when two waves collide they do not pass through each other.

PCNNs have several parameters that may be tuned to considerably modify the outputs. The linking strength, $\beta$, together with the two weight matrices, scales the feeding and linking inputs, while the three potentials, $V$, scale the internal signals. The time constants and the offset parameter of the firing threshold can be exploited to modify the conversions between pulses and magnitudes. The dimension of the convolution kernel directly affects the speed of the autowaves. The dimension of the kernel allows the neurons to communicate with neurons farther away and thus allows the autowave to advance farther in each iteration. The pulse behavior of a single neuron is directly affected by the values of $\alpha_{\Theta}$ and $V_{\Theta}$. The first affects the decay of the threshold value, while the latter affects the height of the threshold increase after the neuron pulses [6].

For each unit, i.e. for each pixel of an image, the PCNNs provide an output value. The time signal $G[n]$, computed by:

$$
G[n]=\frac{1}{N} \sum_{i j} Y_{i j}[n]
$$

is generally exploited to convert the pulse images to a single vector of information. In this way, it is possible to have a global measure of the number of pixels that fire at epoch $[n]$ in a sub-image containing $N$ pixels. The signal associated to $G[n]$ was shown to have properties of invariance to changes in rotation, scale, shift, or skew of an object within the scene [6].

\subsection{Application of PCNN to Toy Examples}

PCNNs have been applied to two toy examples to illustrate the internal activity of the model. Figure 2 shows the first 49 iterations of the algorithm with two images of 150 by 150 pixels. The original inputs $(n=1)$ contain objects with 


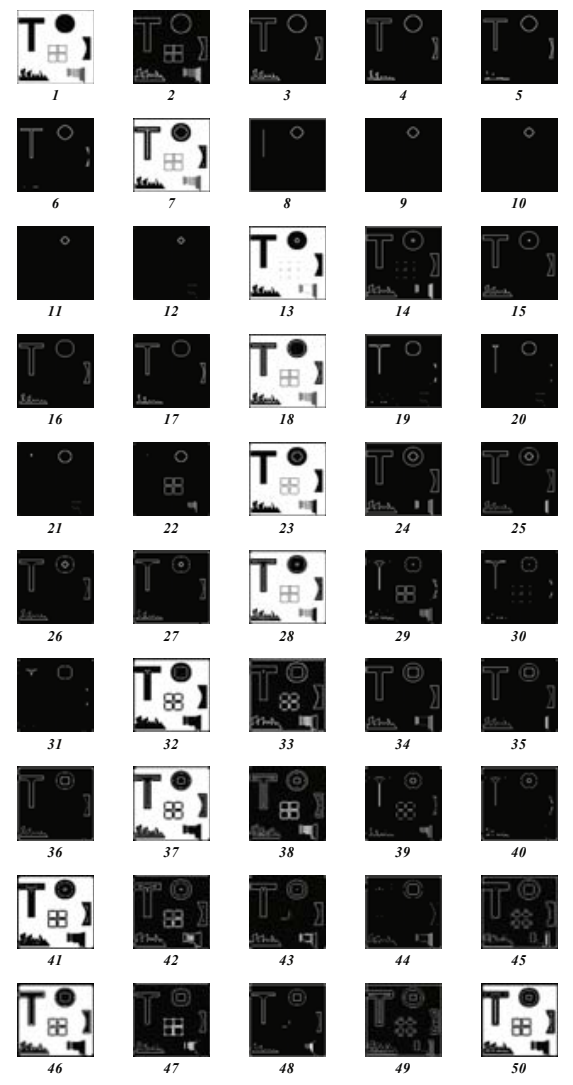

(a)
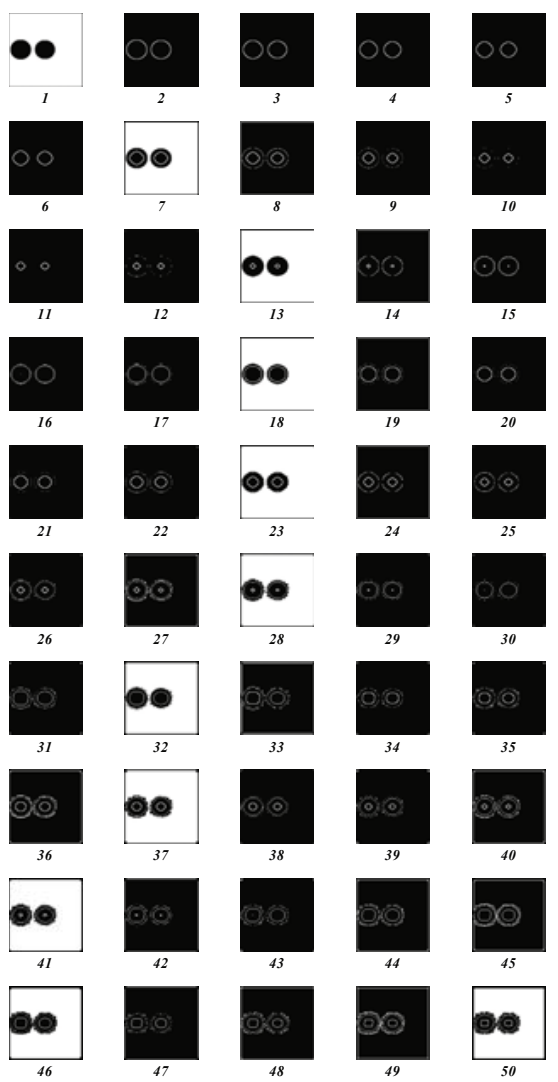

$29 \quad 30$
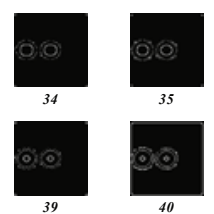

(b)

Fig. 2. Iterations of the PCNN algorithm applied to toy examples of 150 by 150 pixels. As the iterations progress $(n>1)$, the autowaves emanate from the original pulse regions and the shapes of the objects evolve through the epochs due to the pulsing nature of PCNNs.

various shapes, including "T", squares and circles. As the iterations progress $(n>1)$, the autowaves emanate from the original pulse regions and the shapes of the objects evolve through the epochs due to the pulsing nature of PCNNs.

In Figure $3 \mathrm{a}$ and Figure $3 \mathrm{~b}$ are illustrated the progression of the states of a single neuron and trend of $G$ (Equations 1 6) for the toy examples in Figure $2 \mathrm{a}$ and Figure 2 $\mathrm{b}$, respectively. As shown, the internal activity $U$ rises until it becomes larger than the threshold $\Theta$ and the neuron fires $(Y=1)$. Then, the threshold significantly increases and it takes several iterations before the threshold decays enough to allow the neuron to fire again. Moreover, $F, L$ and $U$ maintain values within individual ranges. It is important to note that the threshold $\Theta$ reflects the pulsing nature of the single neuron, while $G$ gives a global measure of the number of pixels that fired at epoch $[n]$. 


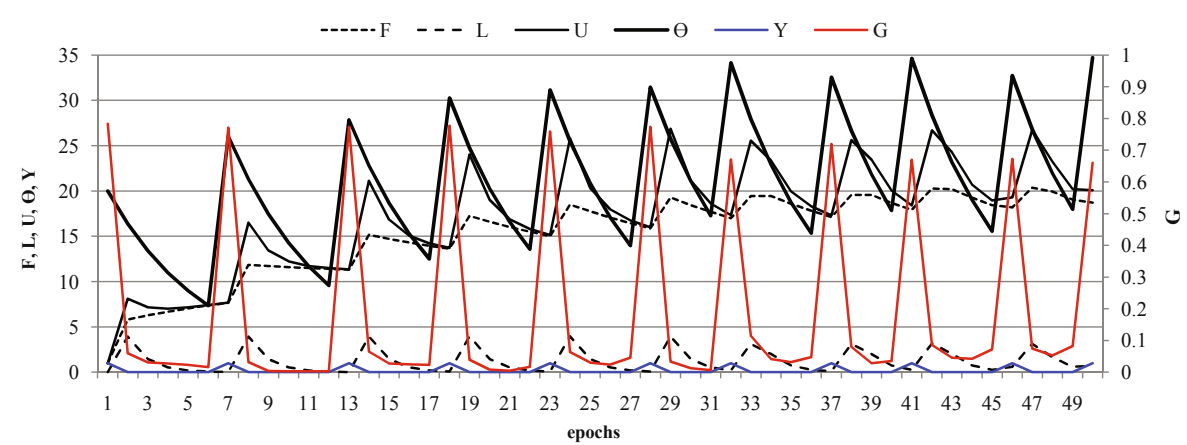

(a)

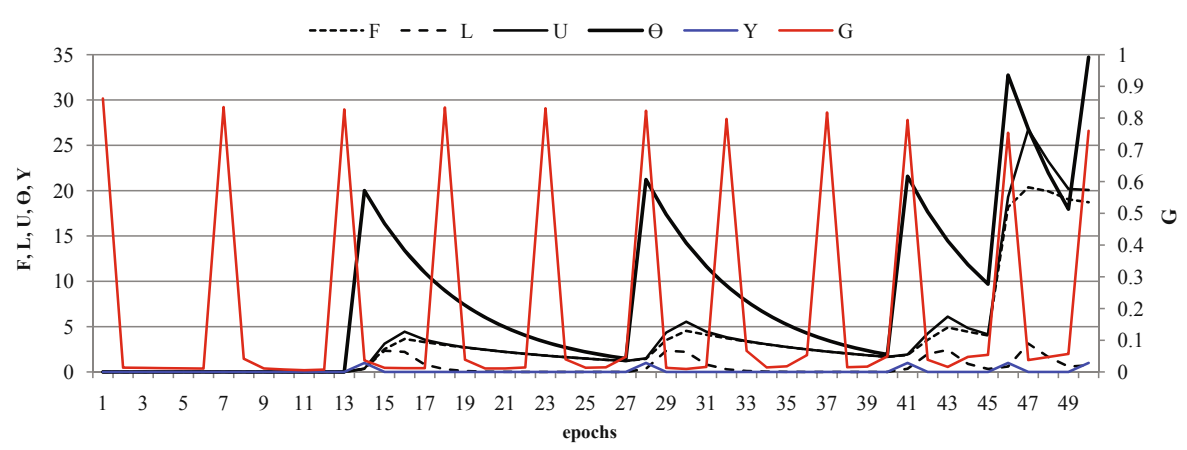

(b)

Fig. 3. Progression of the states of a single neuron (in this example, the central pixel) and trend of $G$ for the toy example in Figure $2 \mathrm{a}$ and Figure 20 respectively

\section{Change Detection with Pulse-Coupled Neural Networks}

The development of fully automatic change detection procedures for very high spatial resolution images is not a trivial task as several issues have to be considered. As discussed in the previous sections, the crucial difficulties include possible different viewing angles, mis-registrations, shadow and other seasonal and meteorological effects which add up and combine to reduce the attainable accuracy in the change detection results. However this challenge has to be faced to fully exploit the big potential offered by the ever-increasing amount of information made available by ongoing and future satellite missions.

PCNNs can be used to individuate, in a fully automatic manner, the areas of an image where a significant change occurred. In particular, the time signal $G[n]$, computed by Equation 6 was shown to have properties of invariance to changes in rotation, scale, shift, or skew of an object within the scene. This last feature makes PCNNs a suitable approach for change detection in very high resolution imagery, where the view angle of the sensor may play an important role. 
In particular, the waves generated by the time signal in each iteration of the algorithm create specific signatures of the scene which are successively compared for the generation of the change map. This can be obtained by measuring the similarity between the time signals associated to the former image and the one associated to the latter. A rather simple and effective way to do this is to use a correlation function operating between the outputs of the PCNNs.

The performance of the algorithm was evaluated on different panchromatic satellite sensors, such as QuickBird and WorldView-1. Qualitative and more quantitative results are reported in the rest of this paper.

\subsection{The Time Signal $G[n]$ in the Multi-spectral Case}

To investigate the time signal $G[n]$ on satellite data, two images $(2825 \times 2917$ pixels) acquired by QuickBird on May 29, 2002 and March 13, 2003 over the Tor Vergata University campus (Rome, Italy) have been exploited. Specifically, multi-spectral images (about $2.4 \mathrm{~m}$ resolution) have been used to have a better comprehension of the PCNN pulsing phase when applied to different bands. In this case, $N=16 \times 16$ pixels.

Four different conditions shown with false colors in Figure 4 have been considered. In Figure $4 \mathrm{a}$ and Figure $4 \mathrm{~b}$ : (UL) big changes, (UR) change in vegetation cover, (DL) small changes and (DR) no-changes. The first area, big changes, represents the construction of a new commercial building. As shown in Figure [5], from the very first epochs the pulsing activity of the two images is relatively different, especially if the waveform is concerned. The change in vegetation cover is illustrated in Figure 5b. During the first few epochs, waveform and time dependence of the two signals appear to be similar. For successive epochs, this correlation decreases, especially due to the well known behavior of near infrared band. The time signal for small changes, i.e. when the changed pixels represent a fraction of sub-image considered, is shown in Figure 5r. During the first epochs, waveforms show slight differences, while the time correlation seems to get lost faster than the previous example. Finally, for the no-changes case shown in Figure 5d, it is possible to note that during the initial epochs both the waveform and the time dependence of the two signals appear to be highly correlated.

Different values can be obtained considering different epoch intervals. This is concisely expressed in Figure 5, where some correlation values obtained considering specific epochs are reported. In particular, it seems not useful to use a high number of epochs since it is not possible to completely distinguish different land changes. On the other hand, the information derived only from the first oscillation (epochs 5-11) appears to be valuable since it allows the discrimination of various land changes.

From this analysis, it seems that PCNNs, once processing an image pair, might be capable to automatically catch those portions where changes occurred. In such a context, an approach based on hot spot detection rather than on changedpixel detection may be more appropriate given the size of the targets (generally buildings) and the huge volume of data archives that it may be necessary to 


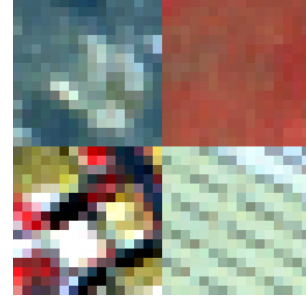

(a)

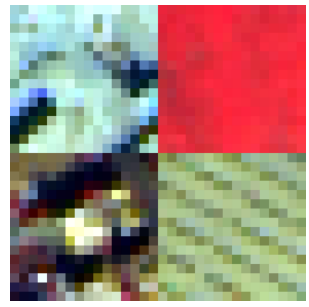

(b)

Fig. 4. Multi-spectral QuickBird images (a) 2002 and (b) 2003 shown with false colors: (UL) big changes, (UR) change in vegetation cover, (DL) small changes and (DR) no-changes

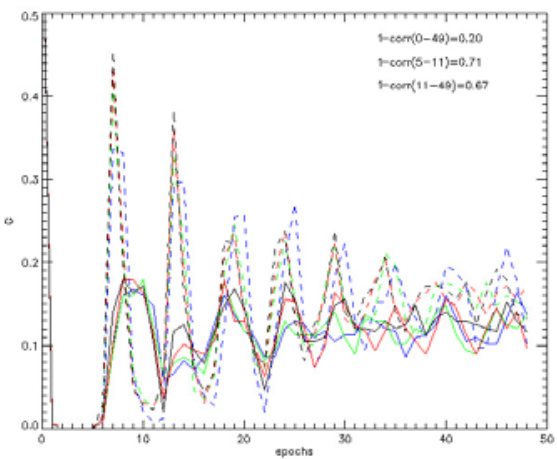

(a)

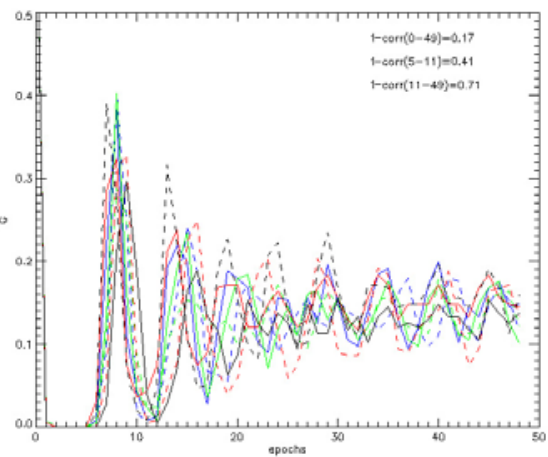

(c)

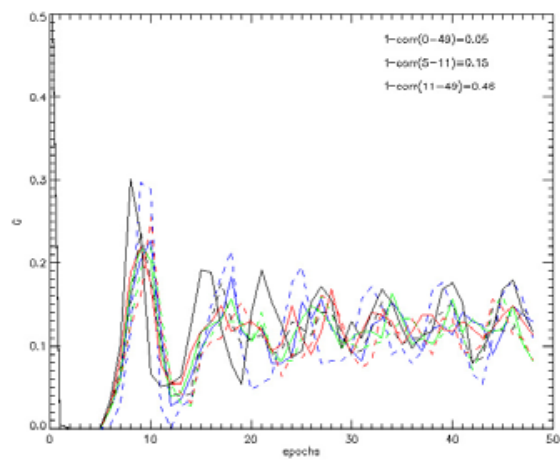

(b)

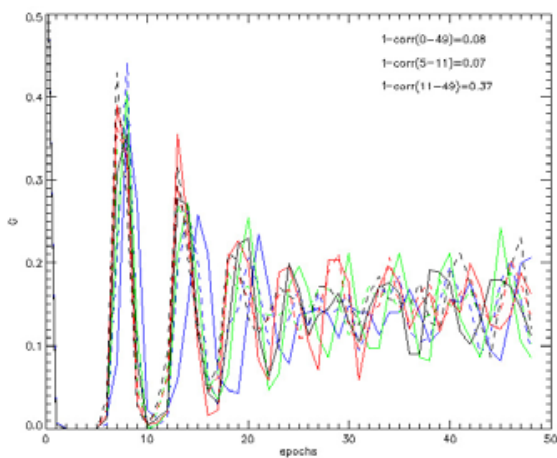

(d)

Fig. 5. The pulsing activity of the two images for the four cases considered in Figure 4. UL case is reported in (a), UR in (b), DL in (c) and DR in (d). Continuous lines represent the 2002 image, while dotted lines correspond to the 2003 image. Red = red channel. Green = green channel, Blue = blue channel, Black = near infrared channel. 
analyze in next future. However, the implementation of a pixel based approach with PCNNs is straightforward, using a window sliding one pixel at the time.

The accuracy of PCNNs in change detection has been evaluated more quantitatively applying PCNNs to the QuickBird imagery of the Tor Vergata University. The panchromatic images have been considered in order to design a single PCNN working with higher resolution $(0.6 \mathrm{~m})$ rather than 4 different ones processing lower resolution $(2.4 \mathrm{~m})$ images. The two panchromatic images are shown in Figure 6] and Figure [b. Few changes occurred in the area during the analyzed time window, the main ones correspond to the construction of new commercial and residential areas. A complete ground reference of changes is reported in Figure 6r. Note that the ground reference included also houses that were already partially built in 2002 .

The size of the PCNN was of 100x100 neurons. For the reasons explained previously, it was preferred only to look for the hot spots where a change could be rather probable. To operate in this way, the PCNN output values were averaged over of the 10,000 neurons belonging to 100x100 boxes. An overlap between adjacent patches of 50 pixels (half patch) was considered. Increasing the overlap would have meant to have more spatial resolution out at the price of an increase of the computational burden. Considering this study was aimed at detecting objects of at least some decades of pixels (such as buildings), an overlapping size of 50 pixels was assumed to be a reasonable compromise. The computed mean correlation value was then used to discriminate between changed and not changed area.

The PCNN result is shown in Figure 6d, while in Figure 6e, for sake of comparison, the image difference result is reported. More in detail, in this latter case, an average value was computed for each box of the difference image and a threshold value was selected to discriminate between changed and not changed areas. In particular, the threshold value was chosen to maximize the number of true positives, keeping reasonably low the number of false positives.

What should be firstly noted is that, at least in this application, the PCNN algorithm did not provide any intermediate outputs, with the correlation values alternatively very close to 0 or 1 . This avoided to search for optimum thresholds to be applied for the final binary response. The accuracy is satisfactory, as 49 out of the 54 objects appearing on the ground reference were detected with no falsealarms. The missed objects are basically structures that were already present in the 2002 image (e.g. foundations or the first few floors of a building), but not completed yet. On the other side, the result given by the image difference technique, although a suitable threshold value was selected, is rather imprecise, presenting a remarkable number of false alarms.

The image shown in Figure 6f has been obtained by a multi-scale procedure. This consists in a new PCNN elaboration, this time on pixel basis, of one of the hot spots generated with the first elaboration. In particular the change area corresponding to the box indicated by the " $*$ " in Figure $6 \mathrm{~d}$. It can be noted that the output reported in Figure 6f is more uniformly distributed within the range between 0 and 1 . Its value has been multiplied with the panchromatic image 


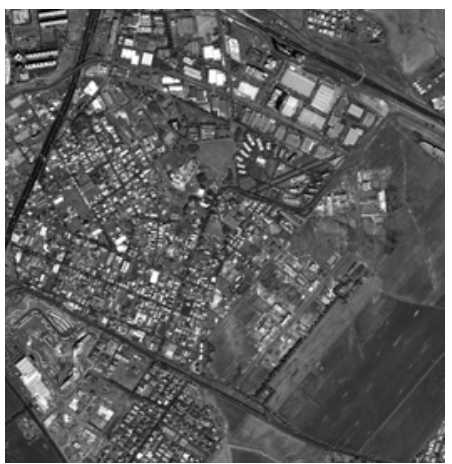

(a)

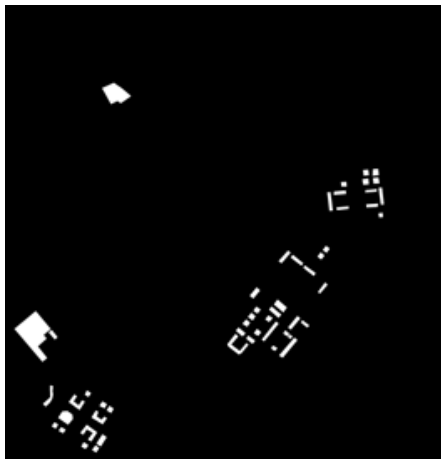

(c)

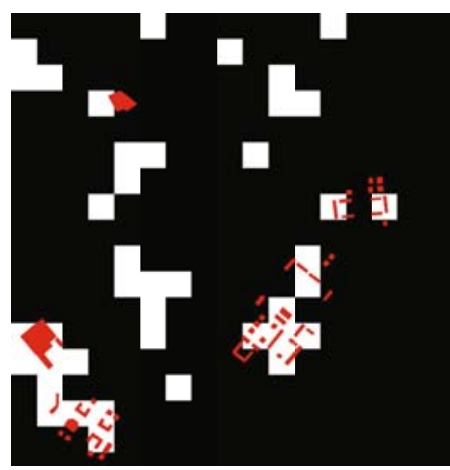

(e)

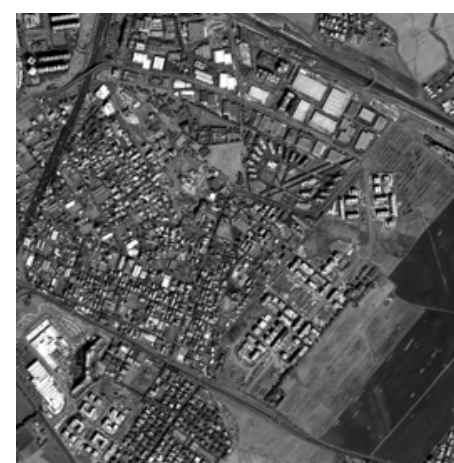

(b)

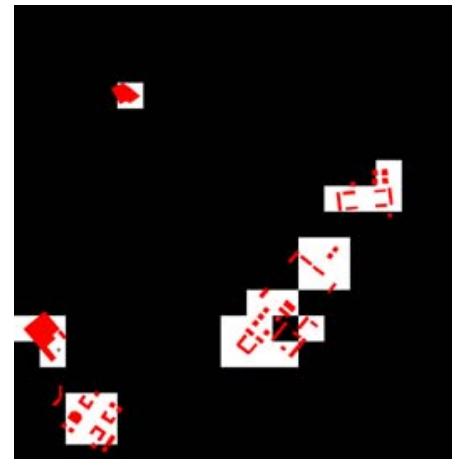

(d)

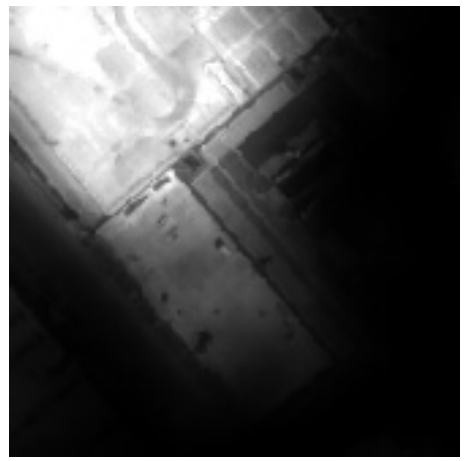

(f)

Fig. 6. Panchromatic image of the Tor Vergata University in (a) 2002 and (b) 2003 and (c) the relative ground reference. Change detection result obtained by: (d) the PCNN elaboration and (e) the standard image difference procedure. In (f) is shown the PCNN pixel-based analysis carried out over one of the previously detected changed areas indicated with " $*$ " in $(d)$. 


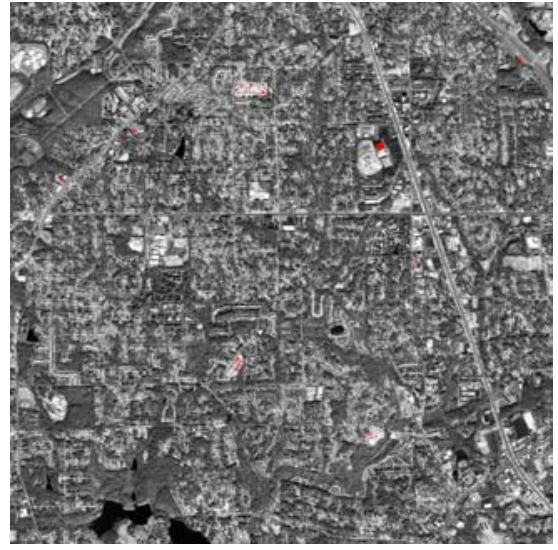

(a)

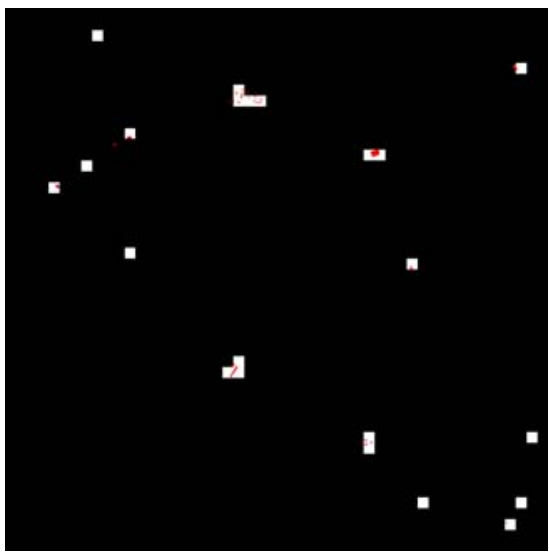

(c)

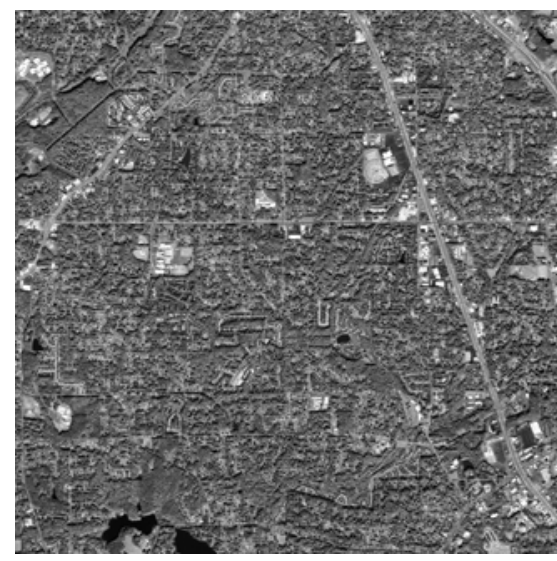

(b)

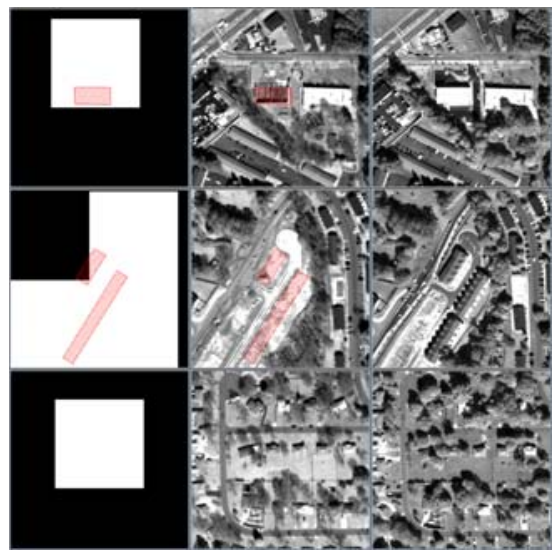

(d)

Fig. 7. QuickBird image with ground reference in red (a) and WorldView-1 image (b) of Atlanta. In (c), the change map provided by PCNN and (d) details of the detected hot spots, including a false alarm.

taken in 2003 to have a result which better exploits the very high resolution property of the original image.

\subsection{Automatic Change Detection in Data Archives}

The study area includes the suburbs of Atlanta, Georgia (U. S. A.). The images were acquired by QuickBird in February 26, 2007 and by WorldView-1 in October 21,2007 for an approximately extension in area of $25 \mathrm{~km}^{2}(10,000 \times 10,000$ pixels). The size of this test case represents an operative scenario where PCNNs give evidence of their potentialities in detecting automatically hot spot areas in data archives. The two images are shown in Figure $7 \mathrm{a}$ and Figure $7 \mathrm{~b}$, respectively. The ground reference of changes is highlighted in Figure $7 \mathrm{a}$ and Figure $7 \mathrm{c}$ in red. 


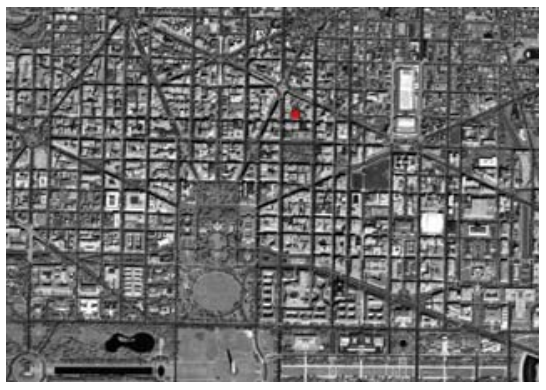

(a)

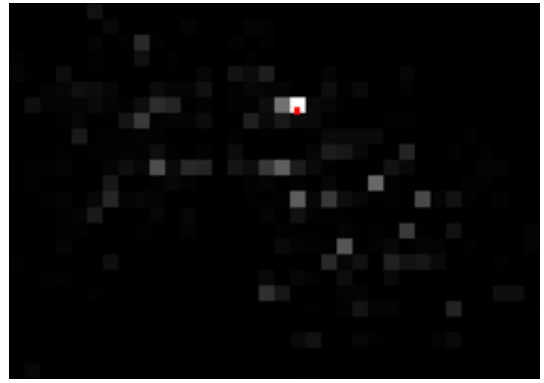

(c)

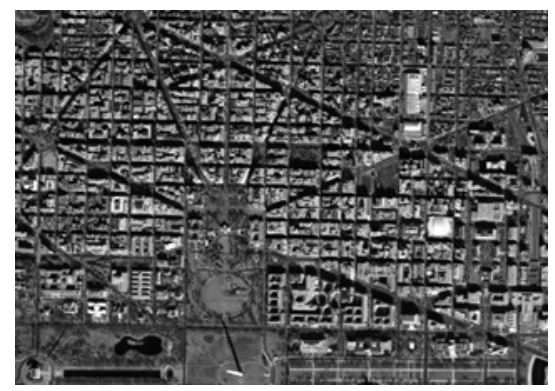

(b)

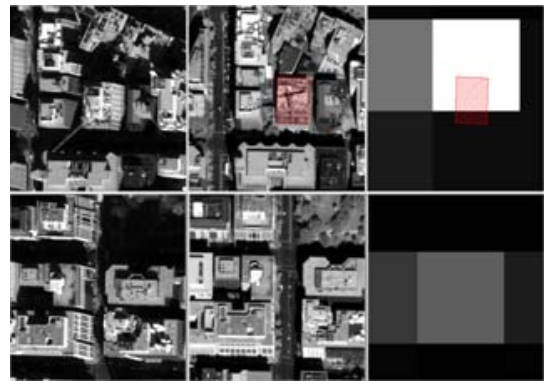

(d)

Fig. 8. QuickBird image with ground reference in red (a) and WorldView-1 image (b) of Washington D. C. In (c), the change map provided by PCNN and (d) details of the detected hot spots, including a false alarm.

Note that the ground reference included also houses that were already partially built during the first acquisition. Many changes occurred although the small time window, mainly corresponding to the construction of new commercial and residential buildings.

As shown in Figure 7f, PCNN confirmed to have good capabilities in the automatic detection of the hot spots corresponding to areas which underwent changes, in this case caused from the construction of new structures. For this test case, where the images have comparable viewing angles, PCNNs did not provide any intermediate outputs, with the correlation values alternatively very close to 0 or 1 . This avoided to search for optimum thresholds to be applied for the final binary response.

The accuracy is satisfactory, as 30 out of the 34 objects appearing on the ground reference were detected with 6 false alarms, mainly due to presence of leaves on the trees in the WorlView-1 image. The missed objects are basically structures that were already present in the first acquisition (e.g. foundations or the first few floors of a building) but not completed yet, or small isolated houses. Details of the detected hot spots (including a false alarm) are shown in Figure $7 \mathrm{~d}$. 


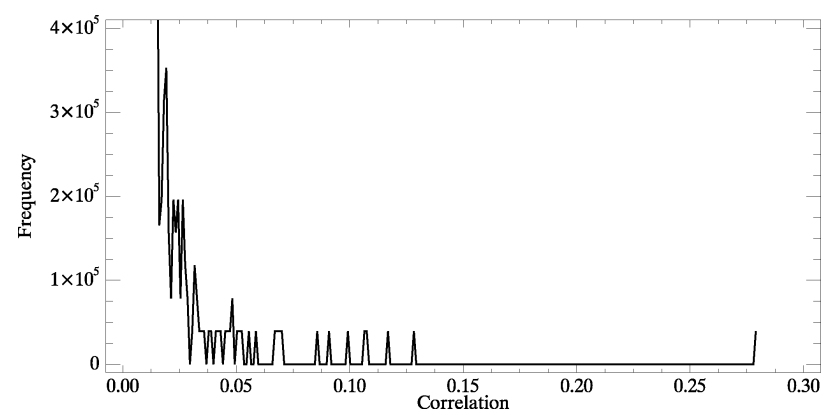

Fig. 9. Frequency of correlation values for the Washington D. C. case. It can be noted that false alarms are characterized by correlation values in the range $(0.00 ; 0.12)$, while the correlation value of the detected hot spot is more than two times higher, i.e. 0.27.

\subsection{Automatic Change Detection in Severe Viewing Conditions}

The study area includes the area of Washington D. C. (U. S. A.). The images were acquired by QuickBird in September 23, 2007 and by WorldView-1 in December 18, 2007 for an approximately extension in area of $9 \mathrm{~km}^{2}(7,000 \times 5,000$ pixels). In this case, the images have been acquired with very different view angles to investigate the performance of PCNNs in this particularly condition. The images are shown in Figure 8, and Figure 8b, respectively. Only one change occurred in the area due the small time window, corresponding to the demolition of a building (highlighted in red in Figure 8a and Figure 85).

As shown in Figure 8f, PCNN detected correctly the only hot spot of change. Differently from the previous case, where values were close to 0 or 1 , non-changed areas show correlation values slightly bigger than 0 . This may be expected due to the very different view angles of the imagery used. For example, the same building is viewed from different directions, occluding different portions of the scene, such as roads or other buildings. However, PCNNs appear to be robust enough to this problem as shown in the plot of Figure 9. Here, on the y-axis, the number of pixels associated to the same measured correlation value is reported. It can be noted that false alarms are characterized by correlation values in the range $(0.00 ; 0.12)$, while the correlation value of the detected hot spot is more than two times higher, i.e. 0.27. Therefore, in this extreme case, the search for an optimum threshold appear to be straightforward. Details of the detected hot spot and an example false alarm are shown in Figure 8], respectively.

\section{Conclusions}

The potential of a novel automatic change detection technique based on PCNNs was investigated. This new neural network model is unsupervised, context sensitive, invariant to an object scale, shift or rotation. Therefore, PCNNs own rather interesting properties for the automatic processing of satellite images. 
The approach aiming at discovering changed subareas in the image (the hot spots) rather than analyzing the single pixel was here preferred. This might be more convenient when large data sets have to be examined, as it should be the case in the very next years when new satellite missions will be providing additional data along with the ones already available.

The application of PCNNs to sub-meter resolution images of urban areas produced promising results. For the Atalanta area, 30 out of the 34 objects appearing on the ground reference were detected with 6 false alarms, mainly due to presence of leaves on the trees in the WorlView-1 image. The goal of the Washington D. C. scene was to demonstrate the robustness of PCNNs when applied to images acquired with very different view angles. Also in this case, the results were satisfactory since false alarms showed less significant correlation values with respect to real changes.

\section{References}

1. Eckhorn, R., Reitboeck, H.J., Arndt, M., Dicke, P.: Feature linking via synchronization among distributed assemblies: simulations of results from cat visual cortex. Neural Computation 2(3), 293-307 (1990)

2. Kuntimad, G., Ranganath, H.S.: Perfect image segmentation using pulse coupled neural networks. IEEE Transactions on Neural Networks 10(3), 591-598 (1999)

3. Gu, X., Yu, D., Zhang, L.: Image thinning using pulse coupled neural network. Pattern Recognition Letters 25(9), 1075-1084 (2004)

4. Karvonen, J.A.: Baltic sea ice sar segmentation and classification using modified pulse-coupled neural networks. IEEE Transactions on Geoscience and Remote Sensing 42(7), 1566-1574 (2004)

5. Waldemark, K., Lindblad, T., Bečanović, V., Guillen, J.L.L., Klingner, P.: Patterns from the sky satellite image analysis using pulse coupled neural networks for preprocessing, segmentation and edge detection. Pattern Recognition Letters 21(3), 227-237 (2000)

6. Lindblad, T., Kinser, J.M.: Image processing using pulse-coupled neural networks. Springer, Heidelberg (2005) 